\title{
A Comprehensive Survey of Web Service Choreography, Orchestration and Workflow Building
}

\author{
K.Adlin Suji \\ MCA Srinivasan Engineering College, \\ Perambalur
}

\author{
S.Sujatha, Ph.D \\ MCA BIT Campus, \\ Anna University, Tiruchirappalli
}

\begin{abstract}
Web services and composition of web services play an important role in building a workflow. This paper discusses the basics of a web service, service composition and methods used for service composition. It also discusses various proposals that were provided as solutions to the problems existing in web service composition. It also proposes future research directions that can efficiently tackle the issues of web service composition.
\end{abstract}

\section{Keywords}

Web mining; web service orchestration; choreography; service composition; ranking and selection; semantic service selection

\section{INTRODUCTION}

Increase in component based systems has lead to the development of Web Services, the inter-operable, platform independent and distributed components that can be used as a part of an application to perform a task. Web Services in general provide the users with a single functionality. The efficient working of any process requires a combination of many such functionalities. Hence we use the process of web service composition. This helps in aggregating the web services in manner that creates a chain of the web services that accomplishes a complete task. This in general is referred to as a workflow. Due to the increase in the number of web services, complexities in the process of selection have also increased. Further, each web service pertaining to a single process has different specifications and properties. Every web service concentrates on providing an efficient solution considering different quality attributes in concern. Hence selection of any web service with respect to the problem in hand is considered the most inefficient way of building a workflow. The quality attributes specified by the consumer must match with the quality attributed concentrated in building up the web service. In this paper, we present a complete analysis of a web service, web service composition methods, problems faced during the construction of web service workflows and future directions of research.

\subsection{Composite Web Services : The Working}

Service-oriented architecture (SOA) is a software design and software architecture design pattern based on discrete pieces of software providing application functionality as services to other applications. This is known as Service-orientation. It is independent of any vendor, product or technology. We use web services to provide a service oriented architecture that help in business process composition.

\subsubsection{Web Services}

The W3C defines a Web service as a software system designed to support interoperable machine-to-machine interaction over a network. It has an interface described in a machine-processable format (specifically WSDL). Other systems interact with the Web service in a manner prescribed by its description using SOAP messages, typically conveyed using HTTP with an XML serialization in conjunction with other Web-related standards [1]. They are self contained modular units that provide certain functionalities to perform a task or a part of a task. It provides a standard format in which applications could interact with other applications irrespective of their operating system, architecture, etc. The functionality of these web services should be described in a format that should be understood by all. This is carried out using WSDL (Web Service Description Language). This description helps in the semantic determination of the web service by the user.

Web services provide fine grained services, while clients require coarse grained, aggregated services. This mapping is performed by the process of web service composition. The process of combining the web services in such a way that it performs sequences of a specific task is called web service composition. Due to the availability of numerous web services, and each satisfying specific QoS properties, an automated composition technique is much more advantageous and accurate when compared to the manual methods. Web service composition can be performed in two different ways:

- A simplistic low-level process modeling and execution languages (like WS-BPEL)

- A complex reasoning process, using high-level unambiguous description language for Web Services (like OWL-S)

Composition of web services can be performed using web service orchestration or web service choreography. Each process has its own positives and negatives. The working of orchestration and choreography is discussed below.

\subsubsection{Web Service Orchestration}

Orchestration usually refers to an executable interorganizational process that is provided by one party $[2,3]$. The executable process has the flexibility to interact with both internal and external web services. Web service orchestration is usually viewed as a method that helps in the composition of multiple web services. Due to the fact that both orchestration and choreography are very similar to each other, process are available that helps in deriving orchestrations from choreographies.

\subsubsection{Web Service Choreography}

Choreography is an integration mechanism that describes message sequences between different parties, and conditions 
and constraints under which messages are exchanged in an inter-organizational business process [2,3,4]. Message interactions between the web services are both local and global. Local interactions deals with communication within a single process, while global interactions take into consideration an overall point of view. Choreographies cannot be self executed [5]. Hence it requires a co-ordination mechanism that helps in performing this process.

In this section we have discussed various basic terminologies and techniques that are required when dealing with the concept of web service selection and composition. Next lets discuss the methods available for service orchestration.

\section{SERVICE COMPOSITION METHODS}

This section describes various methods and pre processing techniques available for efficient web service composition. In general, composition techniques are divided into three categories. Web service ranking and selection mechanisms, workflow design and semantic analysis of web services. Table 1 presents a composition of the web service selection methods.

\subsection{Web Service Ranking and Selection Mechanisms}

Ranking a web service refers to fixing an importance level to it such that the specific web service occupies the highest priority during the next selection request. [6] presents a ranking algorithm working on relationship based measures. This algorithm provides dominance, dominated and dominating score based ranking for the services. Further, request ranking is also provided. It uses the process of approximate skyline and heuristic skyline clustering. The downsides of this process is that there is no optimal match criteria. Reduction of scores will lead to information loss. While fixing the weights requires user preferences, due to the implicit nature of the web services, user feedback is not available. Hence there is a possibility of elimination of good candidates. An efficient method of keyphrase extraction is presented in [7]. Keyphrases help understand information in a document's meta data. Keyphrase extraction is in general considered as ranking problem. [7] uses Multilayer perceptron neural network to perform the process of extraction. Keyword phrase identification is performed using phrase frequency, phrase link and Inverse document frequency. The problem in this method is that ranking training data manually is not possible. Selection of the appropriate number of hidden neurons is not very easily made. Considering too many neurons will lead to an increase in training times, while with inadequate no of neurons, the network cannot model complex data.

A Genetic algorithm based service selection approach was proposed in [9]. It also incorporates the concept of Ant colony optimization, which is a heuristic based optimization approach. The global QoS constraints presented to the system are decomposed into local QoS for further processing. Local constraints are taken in user dimension, hence can be used for large scale multi user service selection. Overall optimal QOS utility within global constraints are used to satisfy user's preference. Service candidate resources are limited, hence web service overload occurs.

[10] presents a globally decentralized and locally centralized architecture for web service orchestration. It is an affinity based scheme, that performs the composition of web services using a central server.
An actor based language unification scheme for orchestration and choreography AB-WSCL is presented in [11]. Messages transfer is carried out in an asynchronous manner, while synchronization is performed among the actors. The activity actor models an activity, while being managed by the WSO (Web Service Orchestration). The relationship between the WSO and WSC (Web Service Choreography) is described here.

An optimal QoS based web service selection scheme is presented in [14]. This is a flexible scheme, that helps service requesters to perform the service selection based on a single QoS and performs QoS based optimization of the service composition. It uses Multi Criteria Decision Making (MCDM) and Integer Programming to provide service composition results. [15] provides a fuzzy based clustering technique for performing the similarity analysis. QoS consensus decision making is performed for efficient results.

A hybrid filtering algorithm for web service filtering recommendation is proposed in [18]. It constructs a cube model that builds relationships between providers, consumers and web services. Collaborative filtering $(\mathrm{CF})$ cannot capture relationships between web services and providers, hence a cube model is used. It contains three matrices; consumer service QOS matrix, provider service binary matrix and consumer provider matrix. It provides web service recommendation to active users and active users are recommended to providers. The two basic process performed in [18] are similarity computation; weight incorporation to solve overestimation and k-nearest neighbor selection that helps in elimination of dissimilar users from top $\mathrm{k}$ neighbors and web services with same provider.

A social harmony search algorithm is proposed in [19]. It helps in the selection of individual web services based on the quality attributes required for making the web service composition. The most important advantage of this method is that it is simple and uses less number of parameters. Stochastic random search is used and the child is generated based on all available values (not just one). Random feasible solutions are initialized and variable generation is performed based on solutions in HM or randomly. Elimination of local optimum is performed. [19] uses normal distributions for updating positions. Functions are designed to perform global search initially and local search in final iterations. Elimination is performed using a constant value.

\subsection{QoS based Workflow Design}

A service selection algorithm that helps in web service composition is discussed in [8]. The LSLO(Local Selection of Local Optimization) based on linear programming. The best ws from each class is selected. Services are ordered based on the score. [8]considers Qos parameters during service selection and optimization and select only the best service for composition. It uses a 3 step process for service selection. Service candidates selection, optimization of best candidate and composition using the best candidates. The advantage of this approach is that only the selected number of QoS parameters are used for analysis, which reduces unnecessary computations to a large extent. It takes up high composition time, which serves as a downside to this approach.

Partitioned web service orchestrations is the process of dividing the centralized web services into fragments, such that the aggregation of these services will provide the functionality that would be provided by the original orchestration. [12] presents an efficient framework for partitioning a web service 
orchestration. This helps in the change propagation from a centralized structure to a distributed structure.

The execution plan formulation for a web service composition is discussed in [13]. The plan formulation also includes the QoS attributes, hence [13] is a QoS aware process that provides maximum QoS performance. User preference takes the highest priority. It is a branch and bound technique, that considers flexible constraints. In its raw form, [13] uses the constraint satisfaction problem as the base logic for performing its operations. The problems in this approach is that decomposing a global optimum into Local Optima that are independent is not a very effective mechanism. There will be dependencies among all QOS parameters like availability, security, cost etc. So we cannot treat them individually as independent elements. [16] presents a probabilistic approach to model and estimate the QoS of workflows.

A genetic based hybrid method for web service selection and composition is proposed in [17]. Dependency \& conflict constraints are considered in this approach. Genetic Algorithm is used for the process of Web Service Selection. Individual elimination from the population is carried out using the local optimization technique. The workflow design consists of all the available implementations of the web service. It constructs an array of web services. All individual web services are considered and infeasible individuals have reduced fitness value. Knowledge based crossover is performed, i.e. concrete web services are selected from parents to form children. Local optimization (optimization of individuals ) is performed in the beginning and end each generation. This method helps increase overall QOS and reduce the number of constraint violations.

A QoS aware multi-granular service composition method is discussed in [20]. It defines behavioral model and proposes a behavioral extracting algorithm. It obtains behavioral signature model from a service composition plan and ensures the best optimal solution. It preserves external observable behavior of composition plan, which can be performed using graph searching technology(DAG). The downside of this approach is that it assumes the need for a granular web service, while the current necessity is for a complete service. Hierarchical business process notation is not common and not practically used. Virtual service class regards only course grained services while ignoring fine grained services.

\subsection{Semantic Analysis of Web Services}

A semantic and QoE based approach for service selection is described in [21]. It provides methods to translate QoE(easy for users) parameters to QOS(easy for service providers)parameters. Provides rules for publishing QoS/QoE parameters. This approach is quite flexible in terms of parameters and QOS expression levels. It uses fixed set of parameters are used to specify quality level. This method does not deal with problem of heterogeneity and it has no standardized SLA/SLS or universally adopted QOS parameters. In real time, several QOS or QOE parameters are available, hence this is not a standardized method, the parameter list keeps expanding. Different NSP's adopt different parameters according to their needs.

A semantic based composition technique is presented in [22]. It helps improve the web's expressability. This process works on overall QoS composition rather than on specific services. It creates a data index table, that maintains three basic details; relationship between data, relationship between data and web services and the QoS offered by the web services. The composition task uses both top down and bottom up approaches. The top down approach is used for the search process and the bottom up approach is used for optimization.

A semantic fault management approach is proposed in [23] which deal with providing composite smart home services. The web service based abstraction layer is used for network service composition in the home area network. Semantic fault management, i.e. diagnosis and correction is performed. BPEL is used for performing the web service orchestration. [24] presents a semantic based method for web service composition and realization. Dynamic composition of web services are performed based on the domain ontology.

\section{Table 1: Web Service Selection Strategies}

\begin{tabular}{|c|c|c|c|}
\hline Ref No & Title & Year & Method Used \\
\hline [8] & $\begin{array}{l}\text { Web Service Selection Algorithm for Dynamic } \\
\text { Service Composition using LSLO Approach }\end{array}$ & 2013 & $\begin{array}{l}\text { - Local selection of local optimization } \\
\text { based on linear programming } \\
\text { - Best web service from each class is } \\
\text { selected } \\
\text { - Linear programming is used }\end{array}$ \\
\hline [9] & $\begin{array}{l}\text { A Hybrid Service Selection Approach for Multi-User } \\
\text { Requests }\end{array}$ & 2012 & $\begin{array}{l}\text { - Genetic Algorithm + Ant colony } \\
\text { algorithm + K-means } \\
\text { - Global QOS constraints are } \\
\text { decomposed to local QOS } \\
\text { - Local constraints are taken in user } \\
\text { dimension } \\
\text { - Overall optimal QOS utility within } \\
\text { global constraints }\end{array}$ \\
\hline
\end{tabular}




\begin{tabular}{|c|c|c|c|}
\hline [17] & $\begin{array}{l}\text { A Hybrid Genetic Algorithm for the Optimal } \\
\text { Constrained Web service Selection Problem in Web } \\
\text { Service } \\
\text { Composition }\end{array}$ & 2010 & $\begin{array}{l}\text { - Hybrid Method } \\
\text { - Dependency \& conflict constraints are } \\
\text { considered } \\
\text { - Genetic Algorithm for Web Service } \\
\text { Selection } \\
\text { - Local optimize to remove individuals } \\
\text { in the population } \\
\text { - Knowledge based crossover }\end{array}$ \\
\hline$[18]$ & $\begin{array}{l}\text { Hybrid Collaborative Filtering algorithm for } \\
\text { bidirectional web service recommendation }\end{array}$ & 2013 & $\begin{array}{l}\text { - Cube model. Relationships between } \\
\text { providers, consumers and web } \\
\text { services } \\
\text { - Three 2D matrixes } \\
\text { 1.consumer service QOS matrix } \\
\text { 2.Provider Service Binary matrix } \\
\text { 3.Consumer Provider matrix }\end{array}$ \\
\hline [19] & $\begin{array}{l}\text { Application of Social Harmony Search Algorithm on } \\
\text { Composite Web Service Selection based on Quality } \\
\text { Attributes }\end{array}$ & 2012 & $\begin{array}{l}\text { - Social harmony search used for } \\
\text { obtaining best web service } \\
\text { - Simple \&less no of parameters } \\
\text { - Uses stochastic random searches } \\
\text { - Elimination of local optimum }\end{array}$ \\
\hline$[20]$ & $\begin{array}{l}\text { QoS-aware and multi-granularity service } \\
\text { composition }\end{array}$ & 2012 & $\begin{array}{l}\text { - Defines behavioral model } \\
\text { - Proposes behavioral extracting } \\
\text { algorithm } \\
\text { - Obtain behavioral signature model } \\
\text { from a service composition plan } \\
\text { - Ensures best optimal solution } \\
\text { - Preserves external observable } \\
\text { behavior of composition plan }\end{array}$ \\
\hline [21] & $\begin{array}{l}\text { A semantic approach for QoS specification of } \\
\text { communication services using QoE parameters }\end{array}$ & 2013 & $\begin{array}{l}\text { Provides methods to translate } \\
\text { QoE(easy for users) parameters to } \\
\text { QOS(easy for service } \\
\text { providers)parameters } \\
\text { - } \text { Provides rules for publishing } \\
\text { QoS/QoE parameters } \\
\text { - Fixed set of parameters are used to } \\
\text { specify quality level } \\
\text { - Do not deal with problem of } \\
\text { heterogeneity } \\
\text { - No standardized SLA/SLS } \\
\text { No universally adopted QOS } \\
\text { parameters }\end{array}$ \\
\hline
\end{tabular}

\section{SERVICE SELECTION PROBLEMS}

Web service selection is the shortlisting of a single or a handful of services that performs the required task satisfying the basic required QoS properties. The description of the process of web service selection is easily said than done. This is because, numerous amount of web services are available in the network and appropriate selection of services that perform the specified task is itself a very complex task, while the QoS parameters add another dimension to the complexity. Selecting a web service that solves the user's problem and provide the required QoS proves to be very difficult. The current selection methods use a user's request and perform the task. This works like an almost tailored search. And other user's requests are not considered during the search process. Hence, as users grow, computation time increases. Too many users requesting the same services would also make the process more tedious. This has lead to the development of a centralized orchestration technique.

Even though this helped in the search process, a centralized orchestration technique itself has some apparent disadvantages. A centralized orchestration will lead to unnecessary traffic, along with inappropriate dependencies. Output confidentiality is reduced. Hence a decentralized architecture was proposed. This architecture faces a few disadvantages such as high cost and weak execution monitoring. Further, the distributed and dynamic nature of the web services will lead to a lot of uncertainties, such as in availability, reliability etc.

Issues faced during a web service paradigm as described in [14] are, Creation of a QoS model, QoS-based service discovery and QoS-based optimization of service 
composition. Further, the QoS described by the consumer might be very different from the providers requirements, even though they might semantically be same. The mapping from consumer to provider is one of the most important issues faced during a service selection process.

A workflow requires interconnection of many such web services. Hence the appropriate service selection problem is magnified. Using any web service in creating the workflow will not be efficient. Each selected service must meet the

QoS requirements specified by the consumer. Further, web services are loosely coupled and there is no centralized middleware for performing the coordination of the services. An efficient web service selection or workflow selection algorithm should be designed to overcome all these issues.

\section{RESEARCH DIRECTIONS}

Web service selection can be performed by using the Mixed Integer Programming method. An integer programming problem is a mathematical optimization or feasibility program in which some or all of the variables are restricted to be integers. Mixed integer linear programming (MILP) involves problems in which only some of the variables, are constrained to be integers. E-commerce negotiations are considered for building the workflow. Analytic Hierarchy Processing (AHP) is used for rating the services according to their QoS parameters. The AHP rating is performed either as pair wise comparison or is directly rated.

The classical approach of Mixed Integer Programming can be superseded by using a Genetic based Approach. Employing a heuristic based approach will help provide much efficient results. Using a GA based approach will further result in obtaining new sequences that have not been discovered earlier. The heuristic based approach can be further optimized by adding a meta heuristic based approach. Optimization of workflow sequences can be carried out by this approach.

Modification of optimization techniques can be used. Parallel programming and transaction based web service selection and processing can be used for further enhancements

\section{CONCLUSION}

The importance of web services, along with the methods of usage of the web services are discussed here. Further, workflow building, which can be decomposed into multiple service selection algorithms is discussed along with the problems encountered during a web service selection process. Available solutions for web service selection is divided into three sections (Ranking and selection of services, workflow design and semantic analysis of web services) and contributions in each section are discussed in detail. It also provides the available research directions for solving the above discussed problems.

\section{REFERENCES}

[1] "Web Services Glossary", February 11, 2004, W3C, Retrieved 2011-04-22.

[2] Mendling, J., Hafner, M., 2008, "From WS-CDL choreography to BPEL process orchestration", Journal of Enterprise Information Management, Vol. 21, No. 5, pp. 525 - 542.

[3] J. Matković,K. Fertalj,2012,"Models for the development of Web service orchestrations",MIPRO 2012, May 2125,Opatija, Croatia.
[4] Kavantzas, N., Burdett, D., Ritzinger, G., Fletcher, T., Lafon, Y.,December 2004,"Web Services Choreography Description Language Version 1.0", W3C Working Draft., Available at: http://www.w3.org/TR/2004/WDws-cdl-10-20041217.

[5] Decker, G., Kopp, O., Leymann, F., Weske, M. 2009,"Interacting services: from specification to execution",Data \& Knowledge Engineering, Vol. 68, no. 10, Elsevier Science Publishers.

[6] Skoutas, D. ; Sacharidis, D. ; Simitsis, A. ; Sellis, T. ,July-Sept. 2010,"Ranking and Clustering Web Services Using Multicriteria Dominance Relationships",IEEE Transactions On Services Computing, Page(s): 163 177.

[7] Kamal Sarkar, Mita Nasipuri and Suranjan Ghose , ,March 2010,"A New Approach to Keyphrase Extraction Using Neural Networks ", IJCSI International Journal of Computer Science Issues, Vol. 7, Issue 2, No 3.

[8] Rathore, M, Suman, U., 2013,"Web Service Selection Algorithm for Dynamic Service Composition using LSLO Approach", Informatics, Electronics \& Vision (ICIEV), 2013 International Conference, ISBN 978-14799-0397-9.

[9] Hua Jin, Hua Zou, Fangchun Yang,Rongheng Lin, Xinchao Zhao,2012,"A Hybrid Service Selection Approach for Multi-User Requests",Embedded Software and Systems (HPCC-ICESS), 2012 IEEE 14th International Conference.

[10] Xi Chen, Huaxin Zeng,Tao Wu,2010,’Decentralized Orchestration with Local Centralized Orchestration for Composite Web Services", The 11th International Conference on Parallel and Distributed Computing, Applications and Technologies, 978-0-7695-4287-4/10 $\$ 26.00$ () 2010 IEEE.

[11] Yong Wang, Xiang Yi, Kai Li, Meilin Liu ,2012,"An Actor-Based Language to Unifying Web Service Orchestration and Web Service Choreography", International Conference on Computer Science and Information Processing (CSIP).

[12] Walid Fdhila, Stefanie Rinderle-Ma, Aymen Baouab, Olivier Perrin, Claude Godart ,2012”On Evolving Partitioned Web Service Orchestrations", IEEE International Conference on Service-Oriented Computing and Applications .

[13] Min Liu a,Mingrui Wanga, Weiming Shena, Nan Luoa, Junwei Yana,2012," A quality of service (QoS)-aware execution plan selection approach for a service composition process",Future Generation Computer Systems 28 (2012) 1080-1089.

[14] Angus F.M. Huang a, Ci-Wei Lan b, Stephen J.H. Yang,2009,“An optimal QoS-based Web service selection scheme",Information Sciences 179 (2009) 3309-3322.

[15] Wei-Li Lin, Chi-Chun Lo, Kuo-Ming Chao , Nick Godwin ,2011,"Multi-group QoS consensus for web services", Journal of Computer and System Sciences 77 (2011) 223-243.

[16] San-Yih Hwang,Haojun Wang , Jian Tang , Jaideep Srivastava ,2007," A probabilistic approach to modeling and estimating the QoS of web-services-based 
workflows", Information Sciences 177 (2007) 54845503.

[17] Maolin Tang, Lifeng Ai,2010,"A Hybrid Genetic Algorithm for the Optimal Constrained Web service Selection Problem in Web Service composition",Evolutionary Computation (CEC), 2010 IEEE Congress On,ISBN 978-1-4244-6909-3.

[18] Jie Cao, Zhiang Wu, Youquan Wang, Yi Zhuang ,Hybrid Collaborative Filtering algorithm for bidirectional web service recommendation, Knowledge and Information Systems (Impact Factor: 2.23). 36(3). DOI: 10.1007/s10115-012-0562-1.

[19] Esfahani, P.M. ， Habibi， J. ; Varaee, T.,2012, "Application of Social Harmony Search Algorithm on Composite Web Service Selection based on Quality Attributes,Genetic and Evolutionary Computing (ICGEC), 2012 Sixth International Conference on,ISBN 978-1-4673-2138-9.

[20] Zaiwen Feng, Rong Peng, Raymond K. Wong, Keqing He, Jian Wang, Songlin Hu, Bing Li, 2013, ’QoS-aware and multi-granularity service composition", Information Systems Frontiers, Volume 15, Issue 4, pp 553-567.

[21] José Cé Júnior, Achilles C. Prudêncio, Roberto Willrich, Madalena P. da Silva ,2013,"A semantic approach for QoS specification of communication services using QoE parameters",Journal of the Brazilian Computer Society, Volume 19, Issue 3, pp 207-221.

[22] Yixin Yan, Bin Xu, Zhifeng Gu, Sen Luo ,2009,"A QoSDriven Approach for Semantic Service Composition", IEEE Conference on Commerce and Enterprise Computing.

[23] Zohar Etzioni, John Keeney, Rob Brennan and David Lewis,2010,"Supporting Composite Smart Home Services with Semantic Fault Management", 978-14244-6949-9/10/\$26.00 @2010 IEEE.

[24] Zhang Hai-tao, Gu Qing-rui, 2010,"A Dynamic Web Services Composition and Realization on the Base of Semantic, 978-1-4244-5824-0/\$26.00 c_2010 IEEE. 